\title{
Overexpression of EPAC2 reduces the invasion of glioma cells via MMP-2
}

\author{
MING JIANG ${ }^{1,2}$, YAN ZHUANG ${ }^{2}$, WANG-CUN ZU ${ }^{3}$, LEI JIAO ${ }^{2}$, SEIDU A. RICHARD ${ }^{2,4,5}$ and SHIMING ZHANG \\ ${ }^{1}$ Department of Neurosurgery, The First Affiliated Hospital of Soochow University, Suzhou, Jiangsu 215006; \\ ${ }^{2}$ Department of Neurosurgery, The Affiliated Hospital of Jiangsu University, Zhenjiang, Jiangsu 212001; \\ ${ }^{3}$ Department of Neurosurgery, Northern Jiangsu People's Hospital, Yangzhou University, Yangzhou, Jiangsu 225000; \\ ${ }^{4}$ Department of Immunology, Jiangsu University, Zhenjiang, Jiangsu 212013, P.R. China; ${ }^{5}$ Department of Medicine, \\ Princefield University, P.O. Box MA 128, Ho, Volta Region, Ghana
}

Received September 28, 2018; Accepted February 26, 2019

DOI: $10.3892 / \mathrm{ol} .2019 .10200$

\begin{abstract}
Exchange proteins directly activated by cAMP (EPACs) are crucial cyclic adenosine 3',5'-monophosphatedetermined signaling pathway intercessors, which are associated with the pathogenesis of neurological disorders and numerous human diseases. To the best of our knowledge, the role of EPAC2 signaling via matrix metalloproteinase 2 (MMP-2) in the pathogenesis of glioma has not been studied. Therefore, the present study focused on the role of EPAC2 in glioma, and assessed the invasiveness of human glioma cell lines following EPAC2 overexpression. Expression levels of EPAC2 in normal brain tissues and clinical glioma specimens were detected by western blotting. An EPAC 2 overexpression vector was transfected into U251 and U87 cell lines to increase the expression levels of EPAC2. Expression levels of MMP-2 were detected by western blotting, and the invasive abilities of glioma cells were detected by a Transwell assay. EPAC2 was relatively highly expressed in normal brain tissue, while EPAC2 expression was significantly decreased in clinical glioma specimens $(\mathrm{P}<0.01)$. In vitro transfection of EPAC2 overexpression vector significantly reduced the MMP-2 protein levels of glioma cells, and, at the same time, the invasive cell number was significantly decreased in a Transwell assay. The present study demonstrated that MMP-2 regulation via EPAC2 overexpression is a novel promising therapeutic route in malignant types of glioma.
\end{abstract}

Correspondence to: Professor Shiming Zhang, Department of Neurosurgery, The First Affiliated Hospital of Soochow University, 188 Shizi Street, Suzhou, Jiangsu 215006, P.R. China

E-mail: shimingzhang@yahoo.com.cn

Key words: exchange protein directly activated by cAMP 1, cyclic adenosine 3',5'-monophosphate, matrix metalloproteinase 2, glioma, overexpression

\section{Introduction}

Gliomas are primary brain tumors formed by the neoplastic transformation of neural stem cells, progenitor cells and differentiated glial cells (astrocyte, oligodendrocyte and ependymal cells) (1). Malignant gliomas are well-known due to their high morbidity, high recurrence, high mortality and low cure rate, with an overall 5-year survival rate of only $13 \%$. Glioma accounts for $\sim 60 \%$ of all types of intracranial primary brain cancer $(2,3)$. Furthermore, these lesions are malignant tumors with multigene features. Their pathogenesis has been suggested to be a result of the triggering of high secretion of proto-oncogenes and/or deletions, as well as inactivation of cancer suppressor genes, triggering idiosyncrasies in cell signaling transmission pathways, modification of the cell cycle, delay of life cycle, and apoptosis, which results in aggressive cell proliferation and malignant alterations $(2,4,5)$.

Exchange proteins directly activated by cAMP (EPACs) are crucial cyclic adenosine 3',5'-monophosphate (cAMP)-determined signaling pathway intercessors that have been associated with the pathogenesis of neurological disorders and numerous human diseases (6). Currently, two isoforms of EPAC have been described; EPAC1 and EPAC2. EPAC2 is a multi-domain protein with a molecular weight of $\sim 116 \mathrm{kDa}$, comprising modulatory and catalytic regions. The amino terminal modulatory region comprises two cyclic nucleotide-binding domains (cNBD-A and cNBD-B) and a disheveled, Egl-10 and pleckstrin (DEP) domain (6,7). cAMP is usually generated from adenosine 5'-triphosphate by adenylate cyclases (8). It is a prototypic second messenger that is involved in cellular responses to numerous triggers, and intermediates signaling pathways associated with a number of human diseases, including cancer, nerve disease, cardiac diseases, diabetes, immunological diseases and urinary dysfunction $(8,9)$.

A previous study demonstrated that cAMP subdues cell growth and p44/42 mitogen-activated protein kinase (MAPK) activities of U87MG glioblastoma cells via the Ras signaling pathway. Furthermore, cAMP blocks p44/42 MAPK action and proliferation in phosphatase and tensin homolog-depleted human glioblastoma cells in vitro via protein kinase A and EPAC stimulation (10). Additionally, a mixture of tricyclic 
antidepressants and a blocker of the purinergic receptor P2Y12 facilitate autophagy-associated cell death in glioma cells via the EPAC subdivision of the cAMP signaling pathway $(11,12)$. Mostafavi et al (13) demonstrated that triggering of the cAMP/EPAC signaling pathway results in an upsurge of connexin 43 secretion in astrocytoma cells. However, isoproterenol stimulates movement of glioma cells in vitro via $\beta 2$-adrenergic receptor/cAMP/EPAC/Rap1B/blockade of $\operatorname{Rac}(11,14)$.

Matrix metalloproteinases (MMPs) are a group of zinc-indomitable endopeptidases that have been implicated in tissue-transformation processes, including wound healing, embryo implantation, tumor invasion, metastasis and angiogenesis (15-18). However, MMP-2 has been reported to be one of four genetic markers that are secreted in glioblastoma multiforme but not anaplastic astrocytoma $(18,19)$. To the best of our knowledge, the effect of EPAC2 on MMP-2 in the pathogenesis of glioma has not been studied. Therefore, in the present study, it was evaluated how in vitro transfection of an EPAC2 overexpression vector may affect MMP-2 protein expression in glioma cells. Additionally, the effects of EPAC2 overexpression on invasive cell numbers in a Transwell assay were evaluated.

\section{Patients and methods}

Clinical samples and cell lines. A total of 10 surgical specimens of patients with clinical glioma (5 males, 5 females; aged, 40-70 years), comprising of 5 low-grade and 5 high-grade, were obtained at the Department of Neurosurgery of The Affiliated Hospital of Jiangsu University (Zhenjiang, China) between March 2017 and November 2017. The specimens comprised glioma tissue and relatively normal brain tissue surrounding the tumor. The normal brain tissues were obtained $2-3 \mathrm{~cm}$ away from the glioma during surgery. Histological examination was carried out to confirm if specimens were normal brain or glioma tissues. No difference (homogeneity) was observed between the normal tissue samples obtained from patients with low and high-grade gliomas. The samples were frozen in liquid nitrogen to preserve them prior to further experiments. The diagnosis of glioma was confirmed by the Pathology Department of The First Affiliated Hospital of Jiangsu University. Classification into low- and high-grade glioma groups was performed. Glioma U251 and U87 cell lines were purchased from Type Culture Collection of the Chinese Academy of Sciences (Shanghai, China). Short tandem repeat profiling was used to authenticate glioma cell lines. The present study was approved by the Ethics Committee of Jiangsu University (Zhenjiang, China), and patients provided written informed consent.

Reagents. Dulbecco's modified Eagle's medium (DMEM; Thermo Fisher Scientific, Inc., Waltham, MA, USA), fetal bovine serum (FBS; Gibco; Thermo Fisher Scientific, Inc.) and trypsin (Sigma-Aldrich; Merck KGaA, Darmstadt, Germany) were obtained. DMEM was supplemented with $10 \% \mathrm{FBS}$, $100 \mathrm{U} / \mathrm{ml}$ penicillin and $100 \mu \mathrm{g} / \mathrm{ml}$ streptomycin. Matrigel basement membrane matrix (BD Biosciences, San Jose, CA, USA) and Transwell chambers (Corning Inc., Corning, NY, USA) were obtained. EPAC2, MMP-2 and $\beta$-actin antibodies were obtained from Abcam (Cambridge, UK). EPAC2 overexpression plasmid was purchased from GenScript (Nanjing, China). Lipofectamine ${ }^{\circledR} 3000$ transfection reagent was obtained from Invitrogen; Thermo Fisher Scientific, Inc.

Glioma U251 and U87 cell cultures. Original glioma U251 and U87 cell lines were washed and minced in $1 \mathrm{X}$ PBS, followed by $0.25 \%$ enzymatic dissociation at $37^{\circ} \mathrm{C}$ for $20 \mathrm{~min}$. Isolated cells $\left(1 \times 10^{6}\right.$ cells $\left./ \mathrm{ml}\right)$ were resuspended in DMEM supplemented with $10 \% \mathrm{FBS}, 100 \mathrm{U} / \mathrm{ml}$ penicillin and $100 \mu \mathrm{g} / \mathrm{ml}$ streptomycin. Subsequently, cells $\left(1 \times 10^{6}\right.$ cells $\left./ \mathrm{ml}\right)$ were seeded in $25 \mathrm{~cm}^{2}$ culture flasks and cultured at $37^{\circ} \mathrm{C}$ in a humidified atmosphere containing $5 \% \mathrm{CO}_{2}$ (Thermo Fisher Scientific, Inc.). The culture medium was replenished every other day. When cells reached $70-90 \%$ confluency, the cells were passaged following $0.25 \%$ trypsin digestion. Single cell cloning was performed at the tenth generation and a stable passage of U251 and U87 cell lines was obtained and validated.

EPAC2 overexpression plasmid transfection of U251 cells and U87 cells. Glioma U251 and U87 cells grown to $70-90 \%$ confluence were transfected with $1 \mathrm{mg} / \mathrm{ml}$ EPAC2 overexpression plasmid (Nanjing KeyGen Biotech Co., Ltd., Nanjing, China) and vector (pcDNA3.1) in complete growth medium, with untransfected U251 and U87 cells as the normal control group. Cells in the plasmid control group were transfected with vector only. Lipofectamine ${ }^{\circledR} 3000$ reagent was diluted at 1:1 ratio in Opti-MEM medium (Gibco; Thermo Fisher Scientific, Inc.) and mixed well. Opti-MEM medium was used to dilute the DNA and to prepare a DNA master mix. Subsequently, P3000TM reagent was added and the solution was mixed thoroughly. Diluted DNA (1:1 ratio) was added to each tube of diluted Lipofectamine ${ }^{\circledR} 3000$ reagent and incubated for $5 \mathrm{~min}$ at room temperature. Then, DNA-liposome complexes were added to the cells. Cells were incubated at $37^{\circ} \mathrm{C}$ for 2 days, followed by extraction of cellular proteins.

Western blotting. Frozen specimens obtained from patients with clinical glioma were lysed with cell lysis buffer for western blotting (Beyotime Institute of Biotechnology, Haimen, China). Protein concentration was quantified using a bicinchoninic acid (BCA) kit (Beyotime Institute of Biotechnology). Total protein extract was added to the loading buffer, $10 \mu \mathrm{g} /$ lane were loaded and separated by SDS-PAGE (12\% gels). Additionally, total protein extract from glioma U251 and U87 cell lines transfected with EPAC2 plasmid and empty plasmid were lysed with cell lysis buffer for western blotting (Beyotime Institute of Biotechnology). Protein concentration was quantified using the BCA kit (Beyotime Institute of Biotechnology). Total protein extracts were added to the loading buffer, $20 \mu \mathrm{g} /$ lane were loaded and separated by $12 \%$ SDS-PAGE. Subsequently, proteins were transferred to a nitrocellulose membrane (Thermo Fisher Scientific, Inc.) at $300 \mathrm{~mA}$ at $4^{\circ} \mathrm{C}$ for $120 \mathrm{~min}$. The membrane was blocked with $5 \%$ skimmed milk at room temperature for $1 \mathrm{~h}$, and incubated overnight at $4^{\circ} \mathrm{C}$ with primary antibodies (1:200 dilution). The primary antibodies used were anti-EPAC2 rabbit monoclonal antibody (mAb; cat. no. ab193665), anti-MMP-2 rabbit $\mathrm{mAb}$ (cat. no. ab181286) and anti- $\beta$-actin mouse $\mathrm{mAb}$ (cat. no. ab6276) all purchased from Abcam). Subsequently, 
the membranes were rinsed three times ( 5 min each), and incubated with secondary antibodies [mouse anti-rabbit immunoglobulin G (IgG)-horseradish peroxidase (HRP; cat. no. sc-2357; Santa Cruz Biotechnology, Inc., Dallas, TX, USA) and rabbit anti-mouse IgG-HRP (cat. no. sc-358914; Santa Cruz Biotechnology, Inc.)] at room temperature for $1 \mathrm{~h}$. The membranes were rinsed, and signal was developed using enhanced chemiluminescence reagents (BeyoECL Star; Beyotime Institute of Biotechnology). Polypeptide bands were detected by an enhanced chemiluminescence detection system (Pierce; Thermo Fisher Scientific, Inc.) and semi-quantified using ImageJ version 1.44p software (National Institutes of Health, Bethesda, MD, USA).

Transwell invasion assays. Transwell chambers (insert diameter, $6.5 \mathrm{~mm}$; pore size, $8.0 \mu \mathrm{m} ; 24$ wells) were coated with $30 \mu \mathrm{l}$ Matrigel (BD Biosciences) for $30 \mathrm{~min}$ at $37^{\circ} \mathrm{C}$. DMEM (600 $\mu \mathrm{l}$ ) containing $10 \% \mathrm{FBS}$ was added to the lower chamber. A total of $100 \mu \mathrm{l}$ cell suspension $\left(1 \times 10^{6} \mathrm{cell} / \mathrm{s} / \mathrm{ml}\right)$ in serum-free medium was added to the upper Transwell chamber. After $24 \mathrm{~h}$ of incubation at $37^{\circ} \mathrm{C}, \mathrm{U} 251$ cells that had invaded across the membrane were stained with $0.1 \%$ crystal violet for $20 \mathrm{~min}$ at room temperature. Non-invasive cells in the upper chamber were gently wiped off with a cotton swab and washed 3 times with PBS. The numbers of invasive cells were observed in five field power magnification under an Olympus CKX31 inverted light microscope (Olympus Corporation, Tokyo, Japan; magnification, $\mathrm{x} 400$ ) and counted. The procedure was repeated for $\mathrm{U} 87$ cells.

Statistical analysis. All experiments were repeated three times. All data are expressed as the means \pm standard deviation. Data were analyzed by unpaired Student's t-test or one-way analysis of variance, followed by Dunnett's post hoc test for comparisons among the control group and multiple dose groups. GraphPad Prism v6.0 (GraphPad Software, Inc., La Jolla, CA, USA) was used to conduct statistical analyses. $\mathrm{P}<0.05$ was considered to indicate a statistically significant difference.

\section{Results}

EPAC2 expression is relatively low in clinical glioma specimens. Expression levels of EPAC2 were relatively low in clinical glioma specimens (low- and high-grade) compared with normal control tissues $(\mathrm{P}<0.01$; Fig. $1 \mathrm{~A}$ and $\mathrm{B})$. Therefore, normal brain tissues expressed more EPAC2 protein compared with clinical glioma tissues, suggesting that the number of EPAC2-secreting brain cells may have decreased during glioma pathogenesis. Furthermore, protein expression levels of EPAC2 in the low-grade clinical glioma samples were higher compared with those in the high-grade clinical glioma samples (Fig. 1A and B). These results suggest that high-grade glioma tissues may secrete less EPAC2 protein levels compared with low-grade glioma tissues. Furthermore, this implied that high-grade clinical gliomas may contain a lower number of EPAC2-secreting brain cells compared with low-grade clinical gliomas.

Transfection of EPAC2 overexpression plasmid into glioma cell lines decreases MMP-2 protein expression. Following
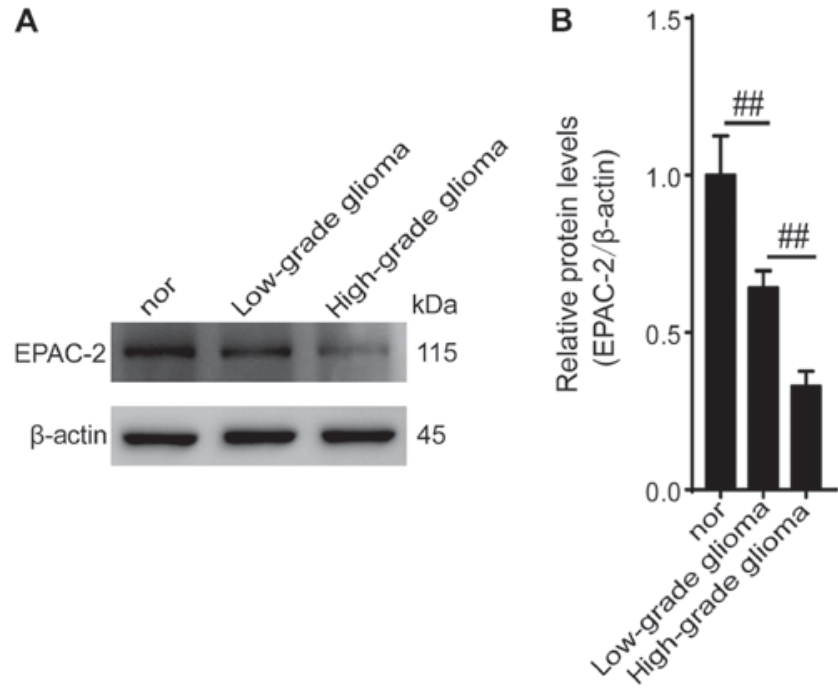

Figure 1. Western blotting. (A) Western blot analysis of EPAC2 protein expression in clinical specimens from patients with glioma (low-grade and high-grade). (B) Analysis revealed that EPAC2 protein levels in glioma specimens (low-grade and high-grade) were significantly lower compared with those in normal brain tissue (obtained from patients with glioma). Higher glioma grade was associated with lower EPAC2 protein levels. Bars represent relative protein levels. The mean values of the protein levels were normalized to $1.0 .{ }^{\# \#} \mathrm{P}<0.01 ; \mathrm{n}=6$. EPAC2, exchange protein directly activated by cAMP 1 ; nor, normal tissue.

transfection of EPAC2 overexpression plasmid (pcDNA3.10) into glioma cell lines (U251 and U87), the protein expression levels of EPAC2 increased significantly $(\mathrm{P}<0.01)$ and the protein expression levels of MMP-2 decreased significantly $(\mathrm{P}<0.01)$. Additionally, the number of invasive cells was significantly decreased in a Transwell assay (Fig. 2A-D). Relative protein expression levels of EPAC2 were elevated in cells transfected with EPAC2 overexpression plasmid compared with the plasmid control U251 cells (Fig. 3A and B). Additionally, in U87 cell lines, relative EPAC2 expression was elevated in cells transfected with EPAC2 overexpression plasmid compared with the normal control and plasmid control cells (Fig. 3D and E).

MMP-2 expression was relatively low in cells transfected with EPAC2 overexpression plasmid compared with the normal control and plasmid control U251 cells (Fig. 3A and C). Additionally, in U87 cells, MMP-2 expression was relatively low in cells transfected with EPAC2 overexpression plasmid compared with the normal control and plasmid control cells (Fig. 3D and F). This suggested that in U251 and U87 cell lines, EPAC2 overexpression may lead to decreased MMP-2 protein levels via the EPAC2/MMP-2 signaling pathway.

\section{Discussion}

Several studies have demonstrated that EPAC2 is secreted predominantly in the brain, pituitary gland, adrenal gland and pancreas (20-22). Seo and Lee (20) demonstrated that EPAC2 is secreted in the cortex, hippocampus and striatum of full-grown mouse brains. Furthermore, previous studies have demonstrated that a reduction in secretion of EPAC2 leads to brain dysfunctions, including alterations in memory and social communication $(20,23,24)$. The present study demonstrated 
A

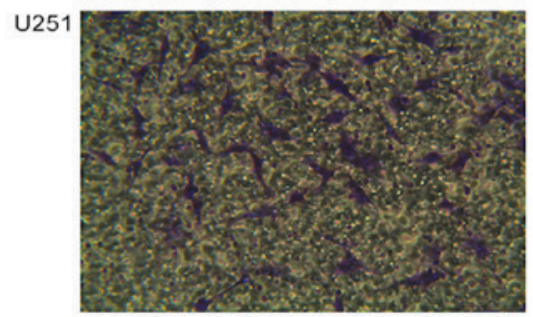

Control

C

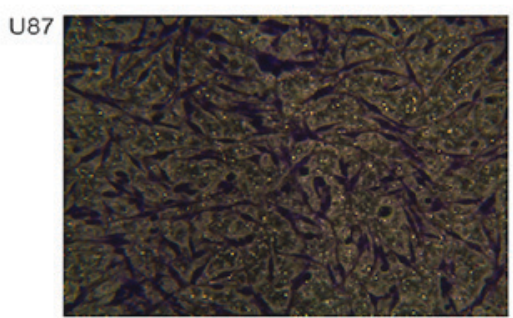

Control

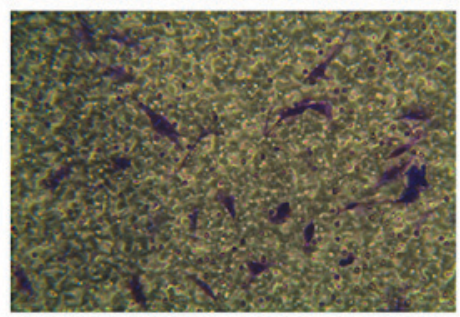

EPAC2-plasmid
B

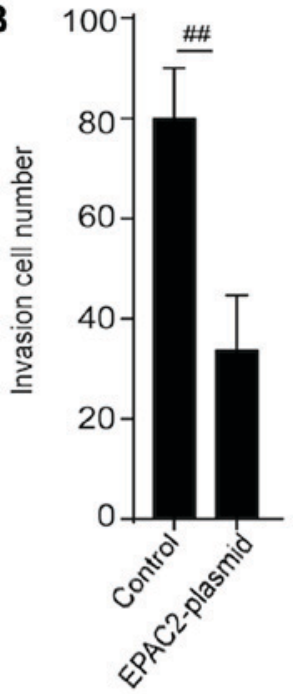

D

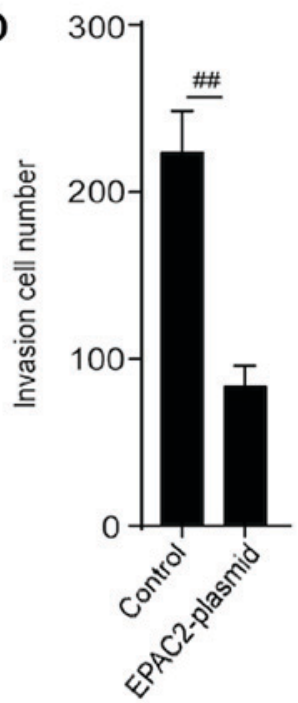

Figure 2. Cell transfection with EPAC2 overexpression plasmid and Transwell invasion assays. (A) U251 cells were transfected with EPAC2 overexpression plasmid and Transwell invasion assays were performed. Counting of purple cells was performed using an Olympus CKX31 inverted light microscope. Original magnification, x100. (B) Numbers of invasive U251 cells were quantified, and the results demonstrated that the invasive cell numbers were significantly reduced following transfection with EPAC2 overexpression plasmid. (C) U87 cells were transfected with EPAC2 overexpression plasmid and Transwell invasion assays were performed. Original magnification, x100. (D) Numbers of invasive U87 cells were quantified, and the results demonstrated that the invasive cell numbers were significantly reduced following transfection with EPAC2 overexpression plasmid. ${ }^{\# \#} \mathrm{P}<0.01$. EPAC2, exchange protein directly activated by cAMP 1 .

that normal brain tissues expressed more EPAC2 levels compared with clinical glioma tissues. These results suggest that the number of EPAC2-secreting cells may decrease during glioma pathogenesis. On the other hand, studies using a global EPAC inhibitor revealed that EPAC modulates astrocyte differentiation via the potassium voltage-gated channel interacting protein 3 transcription factor during neurogenesis $(20,25)$. The present study demonstrated that EPAC2 expression levels in low-grade clinical glioma were higher compared with expression levels in high-grade clinical glioma. This may imply that high-grade clinical glioma tissues contain a smaller number of EPAC2 glands compared with low-grade clinical glioma tissues.

Several studies have demonstrated that MMP-2 and MMP-9 are actively involved in the development of malignant glioma by amplifying cancer cell growth, migration, invasion and angiogenesis $(2,26,27)$. Furthermore, MMP-2 secretion is increased in glioma tissue compared with in normal brain tissue (28-30). Additionally, brain tumors secreting high concentrations of MMP-2 are often associated with high grade, higher invasion, metastasis and angiogenesis (28-30). Studies have demonstrated that there is an increase in MMP-2 expression in cancer and vasculature cells, signifying manifold functions for MMP-2 in cancer development $(30,31)$. Accordingly, further studies identified higher expression levels of MMP-2 in high-grade glioma $(18,27)$. This implies that the higher the grade of glioma, the higher the secretion of MMP-2. Yu et al (15) demonstrated that cancer-derived MMP-2 has two functions during cancer pathogenesis. These functions are: i) Amplification of cancer invasiveness by degrading the extracellular matrix; and ii) amplification of cancer growth by facilitating vessel development and function (15).

The present study demonstrated that, in U251 and U87 cell lines, MMP-2 expression was decreased following 



Figure 3. EPAC2 transfection. (A) U251 cells were transfected with EPAC2 overexpression plasmid to evaluate alterations in MMP-2 protein expression. (B) Statistical analysis following transfection of U251 cells with EPAC2 overexpression plasmid. (C) Statistical analysis suggested that following transfection of U251 cells with EPAC2 overexpression plasmid, the MMP-2 protein levels were significantly decreased. Relative protein expression levels of EPAC2 were elevated in EPAC2-plasmid transfected cells compared with the normal control and plasmid control groups in U251 cells. Bars represent relative protein levels. The mean values of the protein levels were normalized to 1.0. (D) U87 cells were transfected with EPAC2 overexpression plasmid to evaluate alterations in MMP-2 protein expression. (E) Statistical analysis following transfection of U87 cells with EPAC2 overexpression plasmid. (F) Statistical analysis revealed that, following transfection of U87 cell strain with EPAC2 overexpression plasmid, MMP-2 protein levels were significantly decreased. Relative protein expression levels of EPAC2 were elevated in EPAC2-plasmid transfected cells compared with the normal control and plasmid control groups in U87 cells. Bars represent relative protein levels. The mean values of the protein levels were normalized to 1.0 . ${ }^{\# /} \mathrm{P}<0.01$. EPAC2, exchange protein directly activated by cAMP 1; MMP-2, matrix metalloproteinase 2.

EPAC2 overexpression plasmid transfection compared with the normal control and plasmid control cells. These results suggest that EPAC2 overexpression led to decreased MMP-2 protein levels via the EPAC2/MMP-2 signaling pathway in U251 and U87 cell lines. The glioma microenvironment is usually made up of malignant cells as well as normal brain cells. Therefore, in the glioma microenvironment, MMP-2 stimulates EPAC2 glands, which in turn secrete higher levels of EPAC2 during glioma pathogenesis. Seo and Lee (20) established that EPAC2 deficiency impaired proapoptotic caspase adaptor protein (PACAP)-stimulated astrocytic differentiation of neural precursor cells without affecting neuronal differentiation. The authors further indicated that an increase in intracellular calcium levels is fundamental in the PACAP/EPAC2 signaling pathway-stimulated astrocytogenesis (20).

A number of studies have demonstrated that astrocytogenesis is principally associated with the PACAP/EPAC signaling pathway $(25,32)$. Furthermore, EPAC2 peculiarities result in decreased energy metabolism, neurotransmitter, ion concentration regulation and numerous other metabolic activities in nervous tissues $(20,33)$. The aforementioned EPAC2 features result in cognitive dysfunction and psychiatric disorders (20).

A limitation of the present study was the inability to determine other signaling pathways via which EPAC2 expression decreases in glioma specimens besides the EPAC2/MMP-2 signaling pathway. Further studies are required to confirm that increasing EPAC2 levels may have potential therapeutic benefits.

In conclusion, the present study demonstrated that normal brain tissues express more EPAC2 compared with clinical gliomas. Additionally, EPAC2 expression levels in low-grade clinical glioma were relatively high compared with high-grade clinical glioma. Furthermore, in U251 and U87 cell lines, MMP-2 expression was relatively low following EPAC2 overexpression plasmid transfection. This suggested that transfection using EPAC2 overexpression plasmid transfection was able to decrease MMP-2 protein expression levels in U251 and U87 cells (Fig. 4). Therefore, increasing the protein expression 


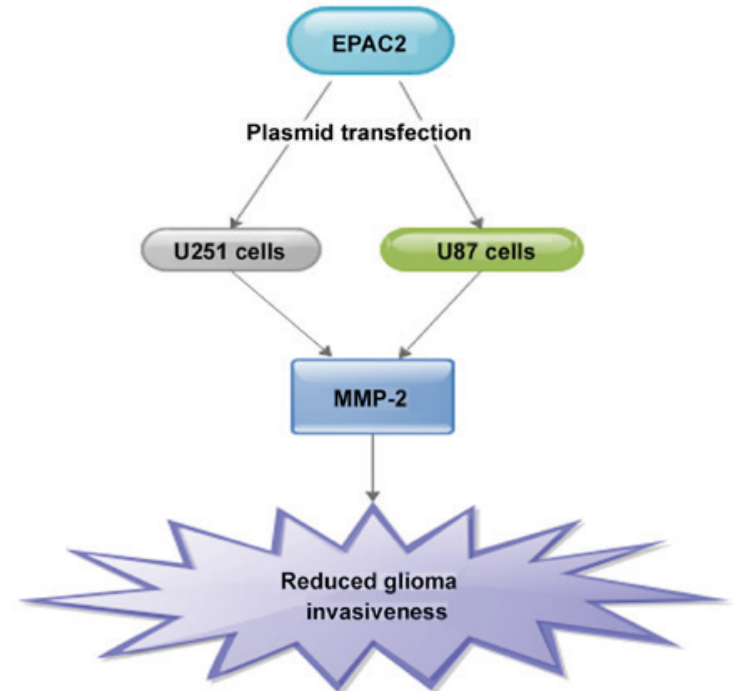

Figure 4. EPAC2-MMP-2 pathway. Diagram showing the EPAC2-MMP-2 pathway which leads to reduced glioma invasiveness. EPAC2, exchange protein directly activated by cAMP 1; MMP-2, matrix metalloproteinase 2 .

levels of EPAC2 could reduce the invasiveness of glioma cells, and provides a novel therapeutic route for malignant glioma.

\section{Acknowledgements}

Not applicable.

\section{Funding}

This study was supported by the Zhenjiang 2017 Science and Technology Innovation Fund (Social Development Project; grant no. SH2017017).

\section{Availability of data and materials}

The datasets used and/or analyzed during the current study are available from the corresponding author on reasonable request.

\section{Authors' contributions}

MJ conceived the study. MJ, YZ, WCZ, LJ, SAR and SZ contributed to data analysis. YZ and SAR drafted the paper, and MJ, YZ, WCZ, LJ, SAR and SZ critically revised the paper. SAR wrote the final paper. All authors agree to be accountable for all aspects of the study.

\section{Ethics approval and consent to participate}

The present study was approved by the Ethics Committee of Jiangsu University, and patients provided written informed consent.

\section{Patient consent for publication}

All patients and their relatives were informed about the intention to publish their data and they provided written informed consent.

\section{Competing interests}

The authors declare that they have no competing interests.

\section{References}

1. Ellison D, Love S, Chimelli L, Harding BN, Lowe JS, Vinter HV, Brandner S and Yong WH: Neuropathology. A reference text of CNS pathology 3rd edition. Edinburgh: Elsevier/Mosby, 2013.

2. Seidu RA, Wu M, Su Z and Xu H: Paradoxical role of high mobility group box 1 in glioma: A suppressor or a promoter? Oncol Rev 11: 325, 2017.

3. Osman MA: Phase II trial of temozolomide and reirradiation using conformal 3D-radiotherapy in recurrent brain gliomas. Ann Transl Med 2: 44, 2014

4. He J, Shan Z, Li L, Liu F, Liu Z, Song M and Zhu H: Expression of glioma stem cell marker CD133 and O6-methylguanine-DNA methyltransferase is associated with resistance to radiotherapy in gliomas. Oncol Rep 26: 1305-1313, 2011.

5. Short SC, Giampieri S, Worku M, Alcaide-German M, Sioftanos G, Bourne S, Lio KI, Shaked-Rabi M and Martindale C: Rad51 inhibition is an effective means of targeting DNA repair in glioma models and CD133+ tumor-derived cells. Neuro Oncol 13: 487-499, 2011.

6. Sugawara K, Shibasaki T, Takahashi H and Seino S: Structure and functional roles of Epac2 (Rapgef4). Gene 575: 577-583, 2016.

7. Bos JL: Epac proteins: Multi-purpose cAMP targets. Trends Biochem Sci 31: 680-686, 2006.

8. Wang P, Liu Z, Chen H, Ye N, Cheng X and Zhou J: Exchange proteins directly activated by cAMP (EPACs): Emerging therapeutic targets. Bioorg Med Chem Lett 27: 1633-1639, 2017.

9. Wild CT, Zhu Y, Na Y, Mei F, Ynalvez MA, Chen H, Cheng X and Zhou J: Functionalized N,N-diphenylamines as potent and selective EPAC2 inhibitors. ACS Med Chem Lett 7: 460-464, 2016.

10. Sugimoto N, Miwa S, Tsuchiya H, Hitomi Y, Nakamura H, Yachie A and Koizumi S: Targeted activation of PKA and Epac promotes glioblastoma regression in vitro. Mol Clin Oncol 1: 281-285, 2013

11. Kumar N, Prasad P, Jash E, Saini M, Husain A, Goldman A and Sehrawat S: Insights into exchange factor directly activated by cAMP (EPAC) as potential target for cancer treatment. Mol Cell Biochem 447: 77-92, 2018.

12. Shchors K, Massaras A and Hanahan D: Dual targeting of the autophagic regulatory circuitry in gliomas with repurposed drugs elicits cell-lethal autophagy and therapeutic benefit. Cancer Cell 28: 456-471, 2015.

13. Mostafavi H, Khaksarian M, Joghataei MT, Soleimani M, Hassanzadeh G, Eftekhari S, Soleimani M, Mousavizadeh K, Estiri H, Ahmadi $S$ and Hadjighassem MR: Selective $\beta 2$ adrenergic agonist increases $\mathrm{Cx} 43$ and miR-451 expression via cAMP-Epac. Mol Med Rep 9: 2405-2410, 2014.

14. Malchinkhuu E, Sato K, Maehama T, Ishiuchi S, Yoshimoto Y, Mogi C, Kimura T, Kurose H, Tomura H and Okajima F: Role of Rap1B and tumor suppressor PTEN in the negative regulation of lysophosphatidic acid-induced migration by isoproterenol in glioma cells. Mol Biol Cell 20: 5156-5165, 2009.

15. Yu CF, Chen FH, Lu MH, Hong JH and Chiang CS: Dual roles of tumour cells-derived matrix metalloproteinase 2 on brain tumour growth and invasion. Br J Cancer 117: 1828-1836, 2017.

16. Richard SA, Sackey M, Su Z and Xu H: Pivotal neuroinflammatory and therapeutic role of high mobility group box 1 in ischemic stroke. Biosci Rep 37: pii: BSR20171104, 2017.

17. Rosenberg GA: Matrix metalloproteinases in brain injury. J Neurotrauma 12: 833-842, 1995.

18. Du R, Petritsch C, Lu K, Liu P, Haller A, Ganss R, Song H, Vandenberg S and Bergers G: Matrix metalloproteinase-2 regulates vascular patterning and growth affecting tumor cell survival and invasion in GBM. Neuro Oncol 10: 254-264, 2008.

19. Zhou YH, Hess KR, Liu L, Linskey ME and Yung WA: Modeling prognosis for patients with malignant astrocytic gliomas: Quantifying the expression of multiple genetic markers and clinical variables. Neuro Oncol 7: 485-494, 2005.

20. Seo $\mathrm{H}$ and Lee K: Epac2 contributes to PACAP-induced astrocytic differentiation through calcium ion influx in neural precursor cells. BMB Rep 49: 128-133, 2016. 
21. Gloerich M and Bos JL: Epac: Defining a new mechanism for cAMP action. Annu Rev Pharmacol Toxicol 50: 355-375, 2010.

22. Grandoch M, Roscioni SS and Schmidt M: The role of Epac proteins, novel cAMP mediators, in the regulation of immune, lung and neuronal function. Br J Pharmacol 159: 265-284, 2010.

23. Lee K, Kobayashi Y, Seo H, Kwak JH, Masuda A, Lim CS, Lee HR, Kang SJ, Park P, Sim SE, et al: Involvement of cAMP-guanine nucleotide exchange factor II in hippocampal long-term depression and behavioral flexibility. Mol Brain 8: 38, 2015.

24. Fernandes HB, Riordan S, Nomura T, Remmers CL, Kraniotis S, Marshall JJ, Kukreja L, Vassar R and Contractor A: Epac2 mediates cAMP-dependent potentiation of neurotransmission in the hippocampus. J Neurosci 35: 6544-6553, 2015.

25. Cebolla B, Fernández-Pérez A, Perea G, Araque A and Vallejo M: DREAM mediates cAMP-dependent, $\mathrm{Ca} 2+$-induced stimulation of GFAP gene expression and regulates cortical astrogliogenesis. J Neurosci 28: 6703-6713, 2008.

26. Guo P, Imanishi Y, Cackowski FC, Jarzynka MJ, Tao HQ, Nishikawa R, Hirose T, Hu B and Cheng SY: Up-regulation of angiopoietin-2, matrix metalloprotease-2, membrane type 1 metalloprotease, and laminin 5 gamma 2 correlates with the invasiveness of human glioma. Am J Pathol 166: 877-890, 2005.

27. Wang M, Wang T, Liu S, Yoshida D and Teramoto A: The expression of matrix metalloproteinase-2 and-9 in human gliomas of different pathological grades. Brain Tumor Pathol 20: 65-72, 2003.
28. Richard SA, Min W, Su Z and Xu HX: Epochal neuroinflammatory role of high mobility group box 1 in central nervous system diseases. AIMS Mol Sci 4: 185-218, 2017.

29. Lampert K, Machein U, Machein MR, Conca W, Peter HH and Volk B: Expression of matrix metalloproteinases and their tissue inhibitors in human brain tumors. Am J Pathol 153: 429-437, 1998.

30. Forsyth PA, Wong H, Laing TD, Rewcastle NB, Morris DG, Muzik H, Leco KJ, Johnston RN, Brasher PM, Sutherland G and Edwards DR: Gelatinase-A (MMP-2), gelatinase-B (MMP-9) and membrane type matrix metalloproteinase-1 (MT1-MMP) are involved in different aspects of the pathophysiology of malignant gliomas. Br J Cancer 79: 1828-1835, 1999.

31. Richard SA, Jiang Y, Xiang LH, Zhou S, Wang J, Su Z and Xu H: Post-translational modifications of high mobility group box 1 and cancer. Am J Transl Res 9: 5181-5196, 2017.

32. Vallejo M: PACAP signaling to DREAM: A cAMP-dependent pathway that regulates cortical astrogliogenesis. Mol Neurobiol 39: 90-100, 2009.

33. Wang DD and Bordey A: The astrocyte odyssey. Progress in neurobiology 86: 342-67, 2008. International (CC BY-NC-ND 4.0) License. 